Journal home page : http://www.sciencedirect.com/science/journal/09977538

Mode coupling instability in friction-induced vibrations and its dependency on system parameters including damping European Journal of Mechanics - A/Solids, Volume 26, Issue 1, January-February 2007, Pages 106-122 Jean-Jacques Sinou and Louis Jézéquel

\title{
MODE COUPLING INSTABILITY IN FRICTION-INDUCED VIBRATIONS AND ITS DEPENDENCY ON SYSTEM PARAMETERS INCLUDING DAMPING
}

\author{
Jean-Jacques SINOU*, Louis JEZEQUEL
}

Laboratoire de Tribologie et Dynamique des Systèmes UMR CNRS 5513

Ecole Centrale de Lyon, 36 avenue Guy de Collongues, 69134 Ecully, France.

\begin{abstract}
Friction-induced vibrations due to coupling modes can cause severe damage and are recognized as one of the most serious problems in industry. In order to avoid these problems, engineers must find a design to reduce or to eliminate mode coupling instabilities in braking systems. Though many researchers have studied the problem of friction-induced vibrations with experimental, analytical and numerical approaches, the effects of system parameters, and more particularly damping, on changes in stableunstable regions and limit cycle amplitudes are not yet fully understood.

The goal of this study is to propose a simple non-linear two-degree-of-freedom system with friction in order to examine the effects of damping on mode coupling instability. By determining eigenvalues of the linearized system and by obtaining the analytical expressions of the Routh-Hurwitz criterion, we will study the stability of the mechanical system's static solution and the evolution of the Hopf bifurcation point as functions of the structural damping and system parameters. It will be demonstrated that the effects of damping on mode coupling instability must be taken into account to avoid design errors. The results indicate that there exists, in some cases, an optimal structural damping ratio between the stable and unstable modes which decreases the unstable region. We also compare the evolution of the limit cycle amplitudes with structural damping and demonstrate that the stable or unstable dynamic behaviour of the coupled modes are completely dependent on structural damping.
\end{abstract}

\section{INTRODUCTION}

Though friction-induced vibration has received considerable attention from a number of researchers [123], there are still no methods which completely eliminate or reduce instabilities. Solving potential friction-induced vibration problems requires a complete understanding and appropriate analysis to identify the effects of all physical parameters on system stability.

For example, it is well known that the stability of the static solution can be affected by the friction coefficient and the associated friction mechanisms. In order to find the most suitable mechanism to describe friction-induced vibration in brake systems, these various mechanisms must be examined. They fall into four categories [1-2]: stick-slip, variable dynamic friction coefficient, sprag-slip [7] and 
geometric coupling of degrees of freedom [8,10,12-16]. The first two approaches rely on changes in the friction coefficient with relative sliding speed affecting system stability. The last two approaches use kinematic constraints and modal coupling in order to develop the instability; in these cases, instability can occur with a constant brake friction coefficient.

Stick-slip is a low sliding speed phenomenon caused when the static friction coefficient is higher than the dynamic coefficient. In 1938, a study by Jarvis and Mills [10] led to an initial understanding that brake squeal was associated with a decrease in friction coefficient with rubbing speed. Due to this negative slope, the steady state sliding becomes unstable and causes friction-induced vibrations. Although this mechanism is still recognised as explaining some low frequency brake vibration problems, it was soon realised that a decrease in friction coefficient was insufficient to explain some friction-induced vibrations. It was later realised that this tribological property was not the only cause of brake squeal, and that vibration could occur when the friction coefficient remained largely constant with speed. Spurr [7] proposed the sprag-slip action which does not depend on a friction coefficient varying with the relative rotation speed of the brake disc. Later researchers (Earles [8, 11-12], North [17], Miller [9], D’Souza and Dweib [18]) developed a more generalised theory describing the mechanism as a geometrically induced or kinematic constraint instability. For example, Jarvis and Mills [16] demonstrated that the variation of the friction coefficient with sliding speed was insufficient to cause friction-induced vibrations and so the instability was due to coupling even if the friction coefficient was constant. It is now accepted that there is no uniform theory for the characterisation of the problem and that stick-slip phenomena [19], negative friction velocity slope [20], sprag-slip phenomena and geometric coupling of the structure involving sliding parts $[7,8,10,12-16]$ contribute to the description of mechanisms causing dynamic instability of brake systems.

The analysis of friction-induced instability is still a vast problem in spite of numerous recent studies on the subject. The stability of the static solution of the mechanical system can be affected by the design and layout of brake components and the effects of all system parameters are not yet clearly understood. This is especially the case for structural damping: in a broad variety of engineering systems, incorporating additional damping into one part of the brake system is undertaken in order to reduce or eliminate significantly friction-induced vibrations. However, it has been observed that the addition of damping to one part of the mechanical system may have a worse effect. Moreover, many studies on mode coupling instability due to friction have relied on undamped model systems. Then, when structural damping is taken into account, its role and effects in the analysis of mode coupling instability is not fully investigated. For example, Earles and Chambers [8] discussed the concept of geometrically induced instability on damped systems and concluded that the effects of damping are too complex to make predictions intuitively and could not be readily anticipated. Recently, Shin et al. [21-22] investigated the effect of damping on a two-degree-of-freedom model and clearly indicated that the amount of damping is a key factor in order to avoid unstable vibrations and stick-slip phenomena. They showed that damped model systems connected through a sliding friction interface can become unstable if damping is added only on one side of the sliding interface. Finally, Hoffmann and Gaul [23] studied the effects of damping on mode-coupling instability in friction-induced oscillations. They also conclude that increasing damping may destabilize friction-induced vibrations $\mathrm{w}$ and that the role of structural damping is not a side effect which can be easily ignored. They show that the effects of damping on mode coupling instability in friction-induced vibrations is a surprising and complex phenomenon.

Given that the influences of structural damping and the associated mode coupling phenomena are not yet fully understood, the goal of this study is to clarify the effects and roles of damping for friction-induced mode coupling instability. For the sake of simplicity a two-degree-of-freedom model will be developed and analysed. One of the biggest advantages of this model is that the stability analysis via the RouthHurwitz criterion can be undertaken and the analytical expressions of the stable/unstable boundary regions can be calculated. The results not only illustrate the effects of damping on the determination of the stable and unstable regions, but also indicate that, in some cases, the most efficient damping ratio between the stable and unstable coupling modes needs to be taken into account to avoid design errors. 
Moreover, mode coupling instability and flutter phenomena will be studied in detail by showing the change in stable and unstable modes due to the variations of structural damping. We will thus demonstrate that stable or unstable dynamic behaviour of the coupled modes are completely dependant on structural damping and that the role of the damping ratio of the coupled modes is essential to the unstable or stable motion of these coupled modes. Then, various parametric studies will be made in order to show the influence of system parameters such as the friction coefficient and the natural frequency ratio between the coupling modes. Finally, the changes in the limit cycle amplitudes for various system parameters will be shown in order to better understand the effects of structural damping on unstable amplitudes.

\section{DESCRIPTION OF THE MECHANICAL MODEL}

Because flutter instability is a mode-coupling phenomenon, a simple self-excited mechanism proposed by Hulten [24-25] will be investigated and developed. This is shown in Figure 1. This model was introduced by Hulten in order to study squeal vibration in drum brakes. For this study, Hulten's model will be sufficient to investigate friction-induced vibration, and to develop analytical expressions in order to better understand the roles and effects of damping. This model is composed of a mass $m$ held against a moving band; the contact between the mass and the band is modelled by two plates supported by two different springs. For the sake of simplicity, we assume that the mass and band surfaces are always in contact. This assumption may be due to a preload applied to the system. The contact can be expressed by two cubic stiffnesses. Damping is integrated as shown in Figure 1. The friction coefficient at contact is assumed to be constant and the band moves at a constant velocity. Then it is assumed that the direction of friction force does not change because the relative velocity between the band speed and $\dot{X}_{1}$ or $\dot{X}_{2}$ is assumed to be positive. All these assumptions are taken into account in order to study a simple non-linear theoretical two-degree-of-freedom system with friction such that the effects of damping on mode coupling instability and the associated analytical developments may be easily investigated.

The tangential force $F_{T}$ due to friction contact is assumed to be proportional to the normal force $F_{N}$ as given by Coulomb's law: $F_{T}=\mu F_{N}$. Assuming the normal force $F_{N}$ is linearly related to the displacement of the mass normal to the contact surface, the resulting equations of motion can be expressed as

$$
\mathbf{M X}+\mathbf{C} \dot{X}+\mathbf{K X}+\mathbf{F N L}(\mathbf{X})=0
$$

with $\mathbf{X}=\left(\begin{array}{ll}X_{1} & X_{2}\end{array}\right)^{T} . \ddot{\mathbf{X}}, \dot{\mathbf{X}}$ and $\mathbf{X}$ are, respectively, the acceleration, velocity, and displacement response 2-dimensional vectors of the degrees-of-freedom. The mass matrix $\mathbf{M}$, the damping matrix $\mathbf{C}$, the stiffness matrix $\mathbf{K}$ and the non-linear vector $\mathbf{F N L}(\mathbf{X})$ are given by

$$
\begin{gathered}
\mathbf{M}=\left[\begin{array}{cc}
m & 0 \\
0 & m
\end{array}\right] \\
\mathbf{C}=\left[\begin{array}{cc}
c_{1} & 0 \\
0 & c_{2}
\end{array}\right] \\
\mathbf{K}=\left[\begin{array}{cc}
k_{1} & -\mu k_{2} \\
\mu k_{1} & k_{2}
\end{array}\right] \\
\mathbf{F N L}(\mathbf{X})=\left(\begin{array}{l}
k_{1}^{N L} X_{1}^{3}-\mu k_{2}^{N L} X_{2}^{3} \\
\mu k_{1}^{N L} X_{1}^{3}+k_{2}^{N L} X_{2}^{3}
\end{array}\right)
\end{gathered}
$$

$\mathbf{K}$ is asymmetric as a result of the friction force. Therefore this system may become unstable.

By dividing these equations by $m$ and considering the relative damping coefficients $\eta_{i}=c_{i} / \sqrt{m_{i} k_{i}}$ $(\mathrm{i}=1,2)$ and natural pulsations $\omega_{0, i}=\sqrt{k_{i} / m_{i}}(\mathrm{i}=1,2)$, the following relations can be established 
where

$$
\left[\begin{array}{ll}
1 & 0 \\
0 & 1
\end{array}\right]\left(\begin{array}{l}
\ddot{X}_{1} \\
\ddot{X}_{2}
\end{array}\right)+\left[\begin{array}{cc}
\eta_{1} \omega_{0,1} & 0 \\
0 & \eta_{2} \omega_{0,2}
\end{array}\right]\left(\begin{array}{l}
\dot{X}_{1} \\
\dot{X}_{2}
\end{array}\right)+\left[\begin{array}{cc}
\omega_{0,1}^{2} & -\mu \omega_{0,2}^{2} \\
\mu \omega_{0,1}^{2} & \omega_{0,2}^{2}
\end{array}\right]\left(\begin{array}{l}
X_{1} \\
X_{2}
\end{array}\right)=\left(\begin{array}{l}
-\varphi_{1}^{N L} X_{1}^{3}+\mu \varphi_{2}^{N L} X_{2}^{3} \\
-\mu \varphi_{1}^{N L} X_{1}^{3}-\varphi_{2}^{N L} X_{2}^{3}
\end{array}\right)
$$

and where $\varphi_{1}^{N L}=k_{1}^{N L} / m$ and $\varphi_{2}^{N L}=k_{2}^{N L} / m$.

The base parameters are given by $\omega_{0,1}=2 \pi \times 1000 \mathrm{rad}_{\mathrm{s}}{ }^{-1} ; \omega_{0,2}=2 \pi \times 800 \mathrm{rad} . \mathrm{s}^{-1} ; \eta_{1}=\eta_{2}=0.02$; $\varphi_{1}^{N L}=\omega_{0,1}^{2}$ and $\varphi_{2}^{N L}=\omega_{0,2}^{2}$.

\section{STABILITY ANALYSIS}

The stability of the static solution is investigated by calculating the eigenvalues $\lambda$ of the linearized system [14-15]. For the non-linear system being studied, the non-linear static solution corresponds to the origin of the system. So the eigenvalues of the linear system can be found by solving the characteristic equation [14-15]

$$
\operatorname{det}\left(\lambda^{2} \mathbf{M}+\lambda \mathbf{C}+\mathbf{K}\right)=0
$$

As long as the real part of all the eigenvalues remains negative, the static solution of the system is stable. When at least one of the eigenvalues has a positive real part, the static solution is unstable. The imaginary part of this eigenvalue represents the frequency of the unstable mode. By considering the friction coefficient $\mu$ as a control parameter, the Hopf bifurcation point is defined by the following conditions:

$$
\begin{aligned}
& \left.\operatorname{Re}\left(\lambda_{\text {center }}(\mu)\right)\right|_{\mathbf{X}=\mathbf{0}, \mu=\mu_{0}}=0 \\
& \left.\operatorname{Re}\left(\lambda_{\text {non-center }}(\mu)\right)\right|_{\mathbf{X}=\mathbf{0}, \mu=\mu_{0}} \neq 0 \\
& \left.\frac{d}{d \mu}(\operatorname{Re}(\lambda(\mu)))\right|_{\mathbf{X}=\mathbf{0}, \mu=\mu_{0}} \neq 0
\end{aligned}
$$

The first condition implies that the system has a pair of purely imaginary eigenvalues $\lambda_{\text {center }}$, while all of the other eigenvalues $\lambda_{\text {non-center }}$ have nonzero real parts at $\left(\mathbf{X}=\mathbf{0}, \mu=\mu_{0}\right)$. The second condition of equation (8), called a transversal condition, implies a transversal or nonzero speed crossing of the imaginary axis.

First, the change in the real and imaginary parts of eigenvalues against the friction coefficient $\mu$ is studied for various damping ratios. Figures 2-3 show the effects of these. As illustrated in Figure 3, the well known behaviour for friction-induced mode coupling is obtained: there are two stable modes at different pulsations when $\mu<\mu_{0}$. As the control parameter $\mu$ increases, these two modes move closer until they reach the bifurcation zone at the Hopf bifurcation point $\mu_{0}$. For $\mu=\mu_{0}$, there is one pair of purely imaginary eigenvalues and all other eigenvalues have negative real parts (as indicated in Figures 2 and 4). After the Hopf bifurcation point $\left(\mu>\mu_{0}\right)$, the two stable and unstable modes couple. As indicated in Figure 2, the real part of the stable and unstable modes (indicated by the stable and unstable branches) are negative and positive, respectively. These effects of damping appear to be very complex. For example, it may be observed that the unstable mode varies due to the damping ratio (see Figure 3): for $\left(\eta_{1}=0.02, \eta_{2}=0.02\right)$, the unstable mode corresponds to the smaller imaginary part of the two coupling modes; for $\left(\eta_{1}=0.02, \eta_{2}=0.005\right)$, the unstable mode corresponds to the larger imaginary part of the two coupling modes.

To further our understanding of the effects of damping, Figure 6 shows the stable and unstable regions of the static solution for various damping ratios $\eta_{1} / \eta_{2}$ (with $\eta_{1}=0.02$ ) and various friction coefficients $\mu$. It clearly appears that increasing damping in only one part of the system may induce mode coupling 
instability. If the damping ratio $\eta_{1} / \eta_{2}$ is very small, the damped system is more unstable than the undamped system (given in Figures 2 and 3); this result shows that analysing the stability of undamped mechanical systems subject to flutter instability may lead to bad designs. So the effects of damping on flutter instability is an important factor to be taken into account to avoid erroneous diagnostics.

As seen in Figure 6, there exists an optimal value of the damping ratio $\left(\eta_{1} / \eta_{2}\right)_{\text {opt }}$ (near 0.8) for which the Hopf bifurcation point $\mu_{0}$ is higher. This result may prove very interesting for obtaining a more stable mechanical system in relation to the structural damping.

Figures 7 and 8 show the change in the real and imaginary parts of eigenvalues versus the damping ratio $\eta_{1} / \eta_{2}$ (with $\eta_{1}=0.02$ ) and various friction coefficients $\mu$. The white surface corresponds to the change in the modes for the static solution that becomes unstable after the Hopf bifurcation point. The black surface is the change in the associated stable static solution. In Figures 8 and 9, it may be observed that the optimal value of the damping ratio $\left(\eta_{1} / \eta_{2}\right)_{\text {opt }} \approx 0.8$ (which corresponds to the more stable system in relation to this damping ratio $\eta_{1} / \eta_{2}$ ) corresponds to the change in the unstable and stable behaviour for the two coupling modes. For $\eta_{1} / \eta_{2}<0.8$, the unstable mode comes from the high pulsation of the two coupling modes; and for $\eta_{1} / \eta_{2}>0.8$, the unstable mode comes from the low pulsation of the two coupling modes.. So, the optimal value of the damping ratio $\eta_{1} / \eta_{2}$ not only defines the most stable mechanical system and the higher value of the Hopf bifurcation point $\mu_{0}$, but also influences the mode coupling behaviour and the origin of the unstable mode

\section{ROUTH-HURWITZ CRITERION}

Throughout the previous computational simulations, it was found that there may exist an optimal value of the damping factor $\eta_{1} / \eta_{2}$ giving a higher Hopf bifurcation point (in relation to the friction coefficient) and so a more stable mechanical system. Because damping appears to be a key factor, we will study its effects on the stability of the static solution of the mechanical system by considering the Routh-Hurwitz criterion. The goal of this development is to obtain the analytical expressions of the Routh-Hurwitz coefficients (in relation to system parameters and more particularly the structural damping) that govern the stability of the static solution of the system.

Considering equation (6)

$$
\operatorname{det}\left(\lambda^{2} \mathbf{M}+\lambda \mathbf{C}+\mathbf{K}\right)=\left|\begin{array}{cc}
\lambda^{2}+\eta_{1} \omega_{0,1} \lambda+\omega_{0,1}^{2} & -\mu \omega_{0,2}^{2} \\
\mu \omega_{0,1}^{2} & \lambda^{2}+\eta_{2} \omega_{0,2} \lambda+\omega_{0,2}^{2}
\end{array}\right|,
$$

we can obtain the $4^{\text {th }}$-order characteristic polynomial

$$
\lambda^{4}+a_{1} \lambda^{3}+a_{2} \lambda^{2}+a_{3} \lambda+a_{4}=0
$$

where $\lambda$ are the eigenvalues of the linearized mechanical system. The expressions $a_{1}, a_{2}, a_{3}$ and $a_{4}$ are given by

$$
\begin{aligned}
& a_{1}=\eta_{1} \omega_{0,1}+\eta_{2} \omega_{0,2} \\
& a_{2}=\eta_{1} \eta_{2} \omega_{0,1} \omega_{0,2}+\omega_{0,1}^{2}+\omega_{0,2}^{2} \\
& a_{3}=\eta_{1} \omega_{0,1} \omega_{0,2}^{2}+\eta_{2} \omega_{0,2} \omega_{0,1}^{2} \\
& a_{4}=\omega_{0,1}^{2} \omega_{0,2}^{2}\left(1+\mu^{2}\right)
\end{aligned}
$$

As explained in the previous section, if all roots of the characteristic equation have a negative real part, the static solution of the system is stable and if at least one root has a positive real part, the static solution is unstable. 
Moreover, applying the Routh-Hurwitz criterion to this characteristic equation gives the four following coefficients

$$
\begin{aligned}
& H_{1}=a_{1} \\
& H_{2}=a_{1} a_{2}-a_{3} \\
& H_{3}=a_{1} a_{2} a_{3}-a_{3}^{2}-a_{4} a_{1}^{2} \\
& H_{4}=a_{1} a_{2} a_{3} a_{4}
\end{aligned}
$$

If all these coefficients are positive, the static solution of the mechanical system is stable. When at least one of the coefficients $H_{i}$ (with $i=1, \ldots, 4$ ) is negative, this static solution is unstable.

Firstly, it may be observed that

$$
\begin{gathered}
H_{1}=\eta_{1} \omega_{0,1}+\eta_{2} \omega_{0,2}>0 \\
H_{2}=\eta_{1} \omega_{0,1}^{3}+\eta_{2} \omega_{0,2}^{3}+\eta_{1} \eta_{2} \omega_{0,1} \omega_{0,2}\left(\eta_{1} \omega_{0,1}+\eta_{2} \omega_{0,2}\right)>0 \\
H_{4}=\omega_{0,1}^{4} \omega_{0,2}^{4}\left(\eta_{1} \omega_{0,1}+\eta_{2} \omega_{0,2}\right)\left(\eta_{1} \eta_{2}+\omega_{0,1} / \omega_{0,2}+\omega_{0,2} / \omega_{0,1}\right)\left(\eta_{1} \omega_{0,2}+\eta_{2} \omega_{0,1}\right)\left(1+\mu^{2}\right)>0
\end{gathered}
$$

Then, the stability of the static solution is governed only by

$$
H_{3}=\omega_{0,1}^{2} \omega_{0,2}^{2}\left(\left(\eta_{1} \omega_{0,1}+\eta_{2} \omega_{0,2}\right)\left(\eta_{1} \eta_{2}+\omega_{0,1} / \omega_{0,2}+\omega_{0,2} / \omega_{0,1}\right)\left(\eta_{1} \omega_{0,2}+\eta_{2} \omega_{0,1}\right)-\left(\eta_{1} \omega_{0,2}+\eta_{2} \omega_{0,1}\right)^{2}-\left(1+\mu^{2}\right)\left(\eta_{1} \omega_{0,1}+\eta_{2} \omega_{0,2}\right)^{2}\right)
$$

So the boundary between the stable and unstable zones and the determination of the Hopf bifurcation point versus the control parameter $\mu_{0}$ is defined by

$$
H_{3}=0=\eta_{1} \eta_{2} \omega_{0,1} \omega_{0,2}\left(\left(\eta_{1} \omega_{0,1}+\eta_{2} \omega_{0,2}\right)\left(\eta_{1} \omega_{0,1} \omega_{0,2}^{2}+\eta_{2} \omega_{0,2} \omega_{0,1}^{2}\right)+\left(\omega_{0,1}^{2}-\omega_{0,2}^{2}\right)^{2}\right)-\omega_{0,1}^{2} \omega_{0,2}^{2} \mu^{2}\left(\eta_{1} \omega_{0,1}+\eta_{2} \omega_{0,2}\right)^{2}
$$

By introducing the following relations

$$
\begin{aligned}
& \alpha_{\omega}=\frac{\omega_{0,1}}{\omega_{0,2}} \\
& \alpha_{\eta}=\frac{\eta_{1}}{\eta_{2}}
\end{aligned}
$$

equation (22) can be rewritten as

$$
0=\omega_{0,2}^{3} \eta_{2}^{2} \alpha_{\omega}\left(\eta_{2}^{2} \sqrt{\alpha_{\omega}} \alpha_{\eta}\left(\sqrt{\alpha_{\omega}} \alpha_{\eta}+1\right)\left(\alpha_{\eta}+\sqrt{\alpha_{\omega}}\right)+\alpha_{\eta}\left(\alpha_{\omega}-1\right)^{2}-\mu^{2} \sqrt{\alpha_{\omega}}\left(\sqrt{\alpha_{\omega}} \alpha_{\eta}+1\right)^{2}\right)
$$

Finally, the value of the Hopf bifurcation point $\mu_{0}$ is given by

$$
\mu_{0}^{2}=\frac{\eta_{2}^{2} \sqrt{\alpha_{\omega}} \alpha_{\eta}\left(\sqrt{\alpha_{\omega}} \alpha_{\eta}+1\right)\left(\alpha_{\eta}+\sqrt{\alpha_{\omega}}\right)+\alpha_{\eta}\left(\alpha_{\omega}-1\right)^{2}}{\sqrt{\alpha_{\omega}}\left(\sqrt{\alpha_{\omega}} \alpha_{\eta}+1\right)^{2}}
$$

In light of this expression, it clearly appears that the value of the Hopf bifurcation point $\mu_{0}$ depends on the structural damping, the damping ratio $\alpha_{\eta}=\frac{\eta_{1}}{\eta_{2}}$, and the natural pulsation ratio $\alpha_{\omega}=\frac{\omega_{0,1}}{\omega_{0,2}}$.

Now, various parametric studies will be undertaken to demonstrate the dependency of the stability of the mechanical system's static solution in relation to the friction coefficient, the structural damping, the damping ratio $\alpha_{\eta}=\frac{\eta_{1}}{\eta_{2}}$, and the natural pulsation ratio $\alpha_{\omega}=\frac{\omega_{0,1}}{\omega_{0,2}}$.

Figure 10 shows the evolution of the Routh-Hurwitz coefficient $H_{3}$ for various values of the friction coefficient $\mu$ and various damping ratios $\eta_{1} / \eta_{2}$ while keeping the structural damping $\eta_{1}$ at its base value $\left(\eta_{1}=0.02\right)$. Moreover, the boundary between the stable and unstable zones is given by using 
equation (26). Results are in perfect correlation with those obtained by determining the eigenvalues of the linearized system (Figure 6).

To further our understanding of the effects of structural damping $\eta_{1}$ and $\eta_{2}$, Figure 11 shows the Hopf bifurcation point $\mu_{0}$ for various damping values $\eta_{1}$ and $\eta_{2}$ by considering equation (27). If the friction coefficient of the mechanical system is higher than the friction coefficient $\mu_{0}$ at the Hopf bifurcation point, the static solution of the system is unstable. It clearly appears that increasing damping in both elements of the system has a beneficial effect and increases the Hopf bifurcation point and therefore the stable region. Moreover, this result suggests that adding damping in only one part of the system may induce mode coupling instability (due to the low Hopf bifurcation point $\mu_{0}$ ).

Figure 12 then illustrates the effects of the structural damping $\eta_{2}$ and damping ratio $\eta_{1} / \eta_{2}$ on the evolution of the Hopf bifurcation point . It may be observed that an optimal damping ratio $\alpha_{\eta}=\eta_{1} / \eta_{2}$ may exist. This optimal ratio depends on the value $\eta_{2}$.

To better assess the influence of the damping ratio $\eta_{1} / \eta_{2}$, Figure 13 shows the effects of varying the damping ratio $\eta_{1} / \eta_{2}$ and pulsation ratio $\omega_{0,1} / \omega_{0,2}$. The general observations from these parametric studies are that for all the values of structural damping $\eta_{2}$ or of the pulsation ratio $\omega_{0,1} / \omega_{0,2}$, the predictions of stable and unstable regions are complex in relation to $\eta_{1} / \eta_{2}$; increasing or decreasing $\eta_{1} / \eta_{2}$ may increase or decrease the Hopf bifurcation point $\mu_{0}$. Then the effects of the damping ratio $\eta_{1} / \eta_{2}$ appear to be a key factor for eliminating mode coupling instability. Moreover, for some values of structural damping $\eta_{2}\left(\eta_{2} \leq 0.9\right)$ or some values of the pulsation ratio $\omega_{0,1} / \omega_{0,2}\left(\omega_{0,1} / \omega_{0,2}<0.9 ; \omega_{0,1} / \omega_{0,2}>1.1\right)$, we note the existence of an optimal value of the damping ratio $\eta_{1} / \eta_{2}$ below which the Hopf bifurcation point is larger and so the system becomes more stable for these parametric conditions.

To further understand the existence of this optimal value of $\eta_{1} / \eta_{2}$, Figure 14 shows its existence in relation to the damping $\eta_{2}$ and the pulsation ratio $\omega_{0,1} / \omega_{0,2}$. The two areas denoted by A define the zones where an optimal value of the damping ratio $\eta_{1} / \eta_{2}$ exists. The area denoted by $\mathrm{B}$ defines the zone where there is no optimal value of $\eta_{1} / \eta_{2}$. In this last case, increasing the structural damping $\eta_{2}$ increases the value of the Hopf bifurcation point $\mu_{0}$ and so increases the stability of the static solution of the mechanical system.

Now, to better assess the values of the optimal structural damping ratio $\eta_{1} / \eta_{2}$ (when it exists), it could be interesting to obtain the analytical expression of this optimal ratio $\eta_{1} / \eta_{2}$ by considering the previous expression (27). The optimal values of $\alpha_{\eta}=\eta_{1} / \eta_{2}$ give the maximal values of $\mu_{0}^{2}$ and so corresponds to one of the solutions of the following expression

$$
\begin{aligned}
& \frac{\partial \mu_{0}^{2}}{\partial \alpha_{\eta}}=0=\frac{\eta_{2}^{2} \sqrt{\alpha_{\omega}} \alpha_{\omega} \alpha_{\eta}^{3}+3 \eta_{2}^{2} \alpha_{\omega} \alpha_{\eta}^{2}+\left(\eta_{2}^{2} \alpha_{\omega}\left(2+\alpha_{\omega}\right)-\sqrt{\alpha_{\omega}}\left(\alpha_{\omega}-1\right)^{2}\right) \alpha_{\eta}+\eta_{2}^{2} \alpha_{\omega}+\left(\alpha_{\omega}-1\right)^{2}}{\sqrt{\alpha_{\omega}}\left(\sqrt{\alpha_{\omega}} \alpha_{\eta}+1\right)^{3}} \\
& \eta_{2}^{2} \sqrt{\alpha_{\omega}} \alpha_{\omega} \alpha_{\eta}^{3}+3 \eta_{2}^{2} \alpha_{\omega} \alpha_{\eta}^{2}+\left(\eta_{2}^{2} \alpha_{\omega}\left(2+\alpha_{\omega}\right)-\sqrt{\alpha_{\omega}}\left(\alpha_{\omega}-1\right)^{2}\right) \alpha_{\eta}+\eta_{2}^{2} \alpha_{\omega}+\left(\alpha_{\omega}-1\right)^{2}=0
\end{aligned}
$$

By introducing the following relation

$$
\alpha_{\eta}=\alpha-\frac{1}{\alpha_{\omega}}
$$

we obtain

$$
\alpha^{3}+p \alpha+q=0
$$

with 


$$
\begin{aligned}
& p=-\frac{3}{\alpha_{\omega}}+\frac{1}{\sqrt{\alpha_{\omega}}}+\frac{2}{\sqrt{\alpha_{\omega}}}-\frac{\left(\alpha_{\omega}-1\right)^{2}}{\eta_{2}^{2} \alpha_{\omega}} \\
& q=\frac{1}{\sqrt{\alpha_{\omega}}}-\frac{1}{\alpha_{\omega}}+\frac{2}{\alpha_{\omega} \sqrt{\alpha_{\omega}}}-\frac{2}{\alpha_{\omega}{ }^{2}}+\frac{2\left(\alpha_{\omega}-1\right)^{2}}{\eta_{2}^{2} \alpha_{\omega} \sqrt{\alpha_{\omega}}}
\end{aligned}
$$

If the discriminant $\Delta=4 p^{3}-27 q^{2}$ is positive, then we have only one real solution given by

$$
\alpha_{1}=\frac{1}{3}(u+v)
$$

and two complex and conjugated solutions given by

$$
\begin{aligned}
& \alpha_{2}=\frac{1}{3}(j u+\bar{j} v) \\
& \alpha_{3}=\frac{1}{3}(\bar{j} u+j v)
\end{aligned}
$$

with

$$
\begin{aligned}
& j=-\frac{1}{2}+i \frac{\sqrt{3}}{2} \\
& u=\sqrt[3]{-\frac{27}{2} q+3 \frac{\sqrt{3}}{2} \sqrt{\Delta}} \\
& v=\sqrt[3]{-\frac{27}{2} q-3 \frac{\sqrt{3}}{2} \sqrt{\Delta}}
\end{aligned}
$$

If the discriminant $\Delta=4 p^{3}-27 q^{2}$ is negative, we have the three real solutions

$$
\begin{aligned}
& \alpha_{1}=\frac{1}{3}(\tilde{u}+\overline{\tilde{u}}) \\
& \alpha_{2}=\frac{1}{3}(j \tilde{u}+\overline{j \tilde{u}}) \\
& \alpha_{3}=\frac{1}{3}\left(j^{2} \tilde{u}+\overline{j^{2} \tilde{u}}\right)
\end{aligned}
$$

with

$$
\tilde{u}=\sqrt[3]{-\frac{27}{2} q+3 i \frac{\sqrt{3}}{2} \sqrt{-\Delta}}
$$

Considering the relations (30-41), the optimal values of $\alpha_{\eta}=\eta_{1} / \eta_{2}$ (corresponding to the maximum of the values of the Hopf bifurcation point) are calculated by varying the structural damping $\eta_{2}$ and the pulsation ratio $\omega_{0,1} / \omega_{0,2}$. Figures 15 and 16 show these optimal values of $\alpha_{\eta}=\eta_{1} / \eta_{2}$ and the associated Hopf bifurcation point $\mu_{0}$, respectively. As explained previously and indicated in Figure 14, for some values of the damping $\eta_{2}$ and the pulsation ratio $\omega_{0,1} / \omega_{0,2}$, this optimal ratio $\alpha_{\eta}=\eta_{1} / \eta_{2}$ does not exist; this is why no optimal value of $\eta_{1} / \eta_{2}$ is given for some parametric conditions in Figure 15.

So, it clearly appears that the optimal value of $\eta_{1} / \eta_{2}$ depends on the structural damping $\eta_{2}$ and the pulsation ratio $\omega_{0,1} / \omega_{0,2}$. Its variation is in the range of $[0.8 ; 3]$ for the considered parametric conditions and so this optimal value is an important factor to be taken into account to avoid poor design. The evolution of associated Hopf bifurcation point appears complex. However, it may be observed that increasing the structural damping $\eta_{2}$ increases the value of the Hopf bifurcation point and so the static solution of the mechanical system is more stable (if the optimal damping ratio $\eta_{1} / \eta_{2}$ is used). Moreover, 
the Hopf bifurcation point decreases when the pulsation ratio $\omega_{0,1} / \omega_{0,2}$ tends towards one, which is easily understood.

By comparing Figures 13 and 15 and considering Figure 12, the damping ratio $\eta_{1} / \eta_{2}$ may be essential to obtain the optimal stable mechanical system. However, in some cases, this damping $\eta_{1} / \eta_{2}$ is not essential (when an optimal value of $\eta_{1} / \eta_{2}$ does not exist) but increasing the structural damping $\eta_{2}$ (or the structural damping $\eta_{1}$ ) makes it possible to increase the stability of the static solution. Finally, if the optimal damping ratio $\eta_{1} / \eta_{2}$ exists, increasing the structural damping $\eta_{2}$ (or the structural damping $\eta_{1}$ ) increases the stability of the static solution.

\section{NON-LINEAR ANALYSIS AND AMPLITUDES}

The determination of the stable and unstable zones is not sufficient for making a better design and the evolution of the limit cycle amplitudes is an important factor to be integrated: the limit cycle amplitudes resulting from various parametric studies may be very small and hence the mode coupling instability could be negligible in these cases.

So, in order to better understand the effect of damping on the limit cycle amplitudes, the non-linear behaviour of the mechanical system and the limit cycles will be calculated by numerically integrating the complete expressions of the non-linear equations of the mechanical system

$$
\left[\begin{array}{ll}
1 & 0 \\
0 & 1
\end{array}\right]\left(\begin{array}{l}
\ddot{X}_{1} \\
\ddot{X}_{2}
\end{array}\right)+\left[\begin{array}{cc}
\eta_{1} \omega_{0,1} & 0 \\
0 & \eta_{2} \omega_{0,2}
\end{array}\right]\left(\begin{array}{l}
\dot{X}_{1} \\
\dot{X}_{2}
\end{array}\right)+\left[\begin{array}{cc}
\omega_{0,1}^{2} & -\mu \omega_{0,2}^{2} \\
\mu \omega_{0,1}^{2} & \omega_{0,2}^{2}
\end{array}\right]\left(\begin{array}{l}
X_{1} \\
X_{2}
\end{array}\right)=\left(\begin{array}{l}
-\varphi_{1}^{N L} X_{1}^{3}+\mu \varphi_{2}^{N L} X_{2}^{3} \\
-\mu \varphi_{1}^{N L} X_{1}^{3}-\varphi_{2}^{N L} X_{2}^{3}
\end{array}\right)
$$

Figures 17 and 18 illustrate the evolution of the maximum amplitudes of the displacements $X_{1}$ and $X_{2}$ for various damping ratios $\eta_{1} / \eta_{2}$ and various friction coefficients $\mu$ (with $\eta_{1}=0.02$ ). First, the stable zones which correspond to amplitudes equal to zero are in perfect correlation with the stability analysis previously conducted.

Second, the well known evolution of the limit cycle amplitudes in relation to the friction coefficient is obtained: increasing the friction coefficient increases the limit cycle amplitudes. Then, it may be observed that increasing the damping ratio $\eta_{1} / \eta_{2}$ may increase or decrease the maximum amplitudes of the displacements. We may then observe that the structural damping $\eta_{2}$ and the damping ratio $\eta_{1} / \eta_{2}$ between the two coupling modes not only influences the stable and unstable regions but also the amplitudes of the non-linear dynamical system. The general observations from Figures 17 and 18 are that the non-linear behaviour of the dynamical system in relation to the evolutions of the structural damping and the friction coefficient may be complex.

Indeed, if the limit cycle amplitudes are estimated near the Hopf bifurcation point $(\mu=0.23)$, the damping ratio $\eta_{1} / \eta_{2}$ influences the non-linear behaviour of the dynamical system and the smaller unstable amplitudes are obtained for the optimal damping ratio which was defined in the previous section and can be shown in Figure 15. If the amplitudes of the displacements $X_{1}$ and $X_{2}$ are calculated for $\mu \leq 0.22$, getting closer to the stable zones decreases the maximum amplitudes of the displacements $X_{1}$ and $X_{2}$. For $\mu>0.24$, it appears that the key factor is not the damping ratio $\eta_{1} / \eta_{2}$ but the value of the structural damping $\eta_{2}$ : increasing the damping ratio $\eta_{1} / \eta_{2}$ (and thus increasing the structural damping $\eta_{1}$ due to the fact that the structural damping $\eta_{2}$ is constant) decreases the maximum amplitudes of the displacements $X_{1}$ and $X_{2}$. From these results, it is clearly shown that if too much damping is added to only one of the coupling modes of the mechanical system, the instability magnitudes may increase. 


\section{CONCLUSION}

This study not only illustrates the effects of system parameters including damping on the determination of stable and unstable zones and on the limit cycle amplitudes, but also indicates that the damping ratio of the coupling modes may be a key factor to be taken into account in order to avoid bad design. Moreover, neglecting damping in a stability analysis or adding damping on only one part of the system may result in worse design and lead to a misunderstanding of the mode coupling instability of mechanical systems. This work indicates that the structural damping $\eta_{1}$ and $\eta_{2}$, the damping ratio $\eta_{1} / \eta_{2}$ and the pulsation ratio $\omega_{0,1} / \omega_{0,2}$ have to be considered in detail in order to design systems with friction-induced oscillations. It has been demonstrated that the Hopf bifurcation point that defines the boundary between the stable and unstable areas depends on the structural damping of the coupling modes, the damping ratio and the pulsation ratio between these two modes. Finally, it was demonstrated that the maximum amplitudes in relation to structural damping is a complex problem and has to be examined in detail in order to avoid bad design.

\section{REFERENCES}

1 R.A. Ibrahim, Friction-Induced Vibration, Chatter, Squeal and Chaos: Part I - Mechanics of Contact and Friction, ASME Applied Mechanics Review, 47(7) (1994) 209-226.

2 R.A. Ibrahim, Friction-Induced Vibration, Chatter, Squeal and Chaos: Part II - Dynamics and Modeling, Applied Mechanics Review, ASME Applied Mechanics Review, 47(7) (1994) 227-253.

3 B. Armstrong-Hélouvry, P. DuPont, C. Canudas de Wit, A survey of models, analysis tools and compensation methods for the control of machines with friction, Automatica 30 (7) (1994) 10931138.

4 D.A. Crolla, A.M. Lang, Brake Noise and Vibration - State of Art. Proceedings of the $17^{\text {th }}$ LeedsLyon Symposium on Tribology, 18(1991) 165-174.

5 J.T. Oden, J.A.C. Martins, Models and computational methods for dynamic friction phenomena, Computer Methods in Applied Mechanics and Engineering 52 (1985) 527-634.

6 N.M. Kinkaid, O.M. O’Reilly, P. Papadopoulos, Automotive disc brake squeal, Journal of Sound and Vibration 267 (2003) 105-166.

7 R.T. Spurr, A theory of brake squeal, Proc. Auto. Div., Instn. Mech. Engrs, 1 (1961) 33-40.

8 S.W.E. Earles, P.W. Chambers, Disc brake squeal noise generation: predicting its dependency on system parameters including damping. International Journal of Vehicle Design 8(1987) 538-552.

$9 \quad$ N. Millner, An Analysis of Disc Brake Squeal. SAE Paper 780332 (1978).

10 R.P. Jarvis, B. Mills, Vibrations induced by dry friction. Proc. Instn. Mech. Engrs, 178 (1963/1964) 847-866.

11 S.W.E. Earles, G.B. Soar, Squeal noise in disc brakes. Proc.I.Mech.E.Conf. on Vibration and Noise in Motor Vehicles (1971) 61-69.

12 S.W.E Earles and C.K. Lee, Instabilities arising from the frictional interaction of a pin-disc system resulting in noise generation. Trans ASME, J. Engng Ind., 98(1) (1976) 81-86.

13 P. Chambrette, L. Jézéquel, Stability of a beam rubbed against a rotating disc. European Journal of Mechanics, A/Solids 11(1992) 107-138.

14 J-J. Sinou, F. Thouverez, L. Jézéquel, Stability and non-linear analysis of a complex rotor/stator contact. Journal of Sound and Vibration 278(2004) 1095-1129.

15 J.J. Sinou, F. Thouverez, L. Jézéquel, Analysis of friction and instability by the centre manifold theory for a non-linear sprag-slip model, Journal of Sound and Vibration 265 (2003) 527-559.

16 R.P. Jarvis and B.Mills. Vibrations induced by dry friction. Proc. Instn. Mech. Engrs, 178(32) (1963/1964) 847-866.

17 M.R. North, A Mechanism of disc brake squeal. 14 ${ }^{\text {th }}$ FISITA congress, Paper 1/9. (1972). 
18 A.F. D'Souza and A.H. Dweib. Self-excited Vibrations induced by Dry Friction. Part 2: Stability and Limit-cycle Analysis. Journal of Sound and Vibration, 137(2) 1990 177-190.

19 S.S. Antoniou, A. Cameron and C.R. Gentle. The Friction-speed Relation from Stick-Slip Data Wear, 36 (1976) 235-254.

20 C. Gao, D. Kuhlmann-Wilsdorf and D.D. Makel. The Dynamic Analysis of Stick-Slip. Wear, 173 (1994) 1-12.

21 K. Shin, J-E. Oh, M.J. Brennan, Nonlinear analysis of friction induced vibrations of a two degree of freedom model for disc brake squeal noise. JSME International Journal 45(2002) 426-432.

22 K. Shin, M.J. Brennan J-E. Oh, C.J. Harris, Analysis of disc brake noise using a two-degree-offreedom model. Journal of Sound and Vibration 254(2002) 837-848.

23 N. Hoffmann, L. Gaul, Effects of damping on mode-coupling instability in friction-induced oscillations. ZAMM · Z. Angew. Math. Mech. 83(8) (2003) 524-534.

24 J. Hultén, Drum Brake Squeal - A Self-Exciting Mechanism with Constant Friction, SAE Truck and Bus Meeting, 1993, Detroit, Michigan, USA, SAE Paper 932965 (1993).

25 J. Hultén, Friction Phenomena Related to Drum Brake Squeal Instabilities, ASME Design Engineering Technical Conferences, Sacramento, California, ASME Paper DETC97/VIB-4161 (1997).

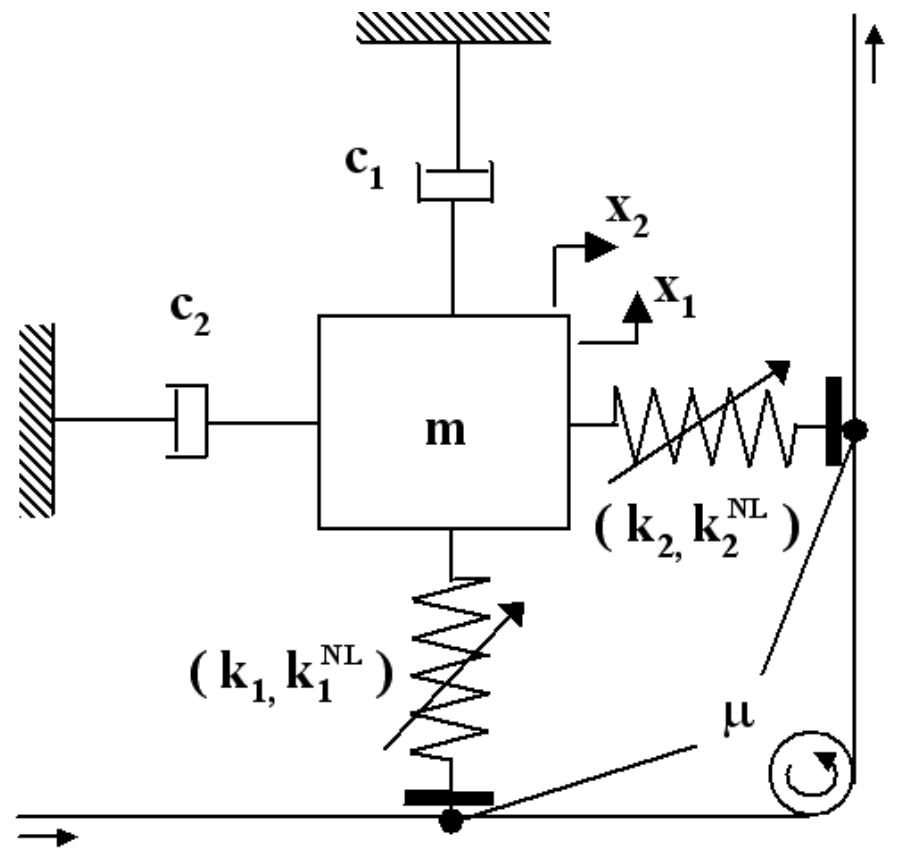

7. Figure $1:$ mechanical model 


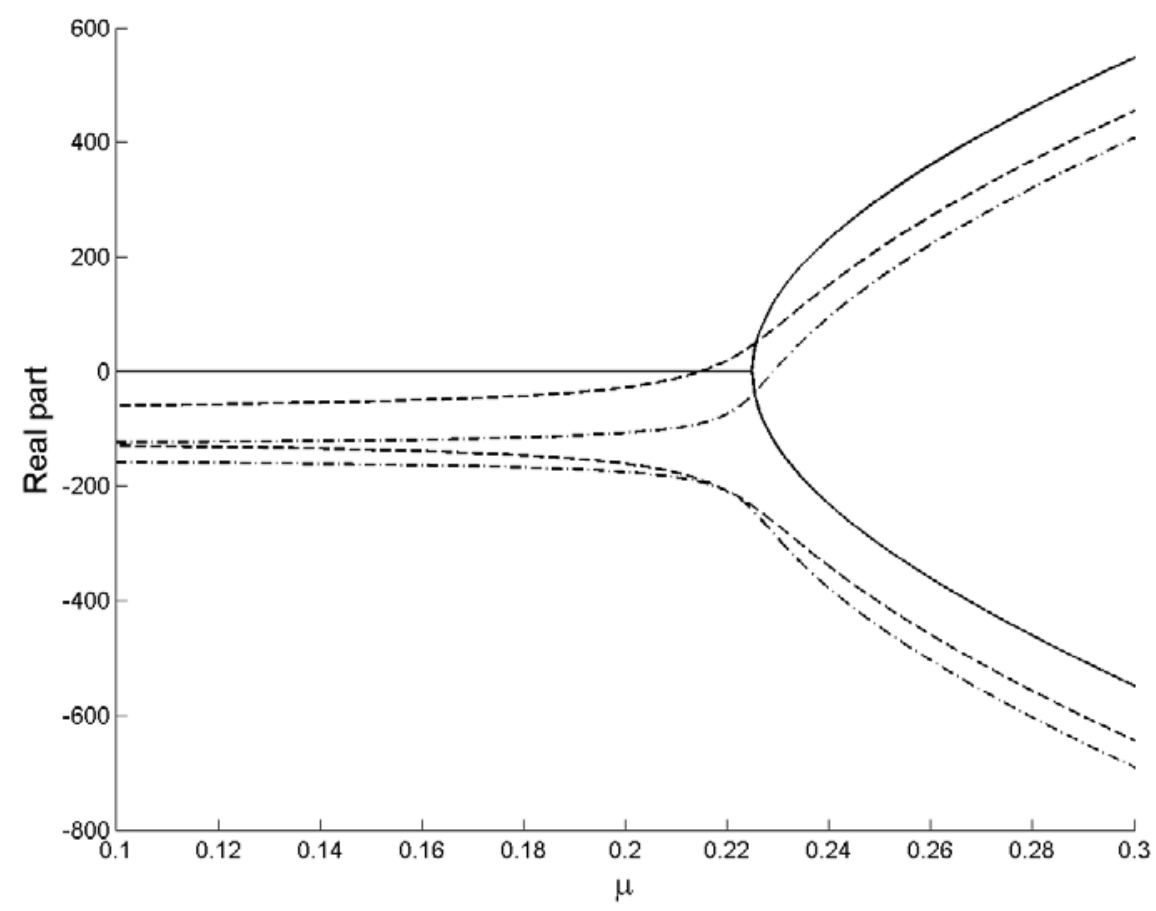

Figure 2 : Evolution of the real part of eigenvalues against the friction coefficient $\mu$ for various proportional structural damping

$\left(-\eta_{1}=\eta_{2}=0----\eta_{1}=0.02, \eta_{2}=0.05 \quad-\cdot-\eta_{1}=\eta_{2}=0.05\right)$

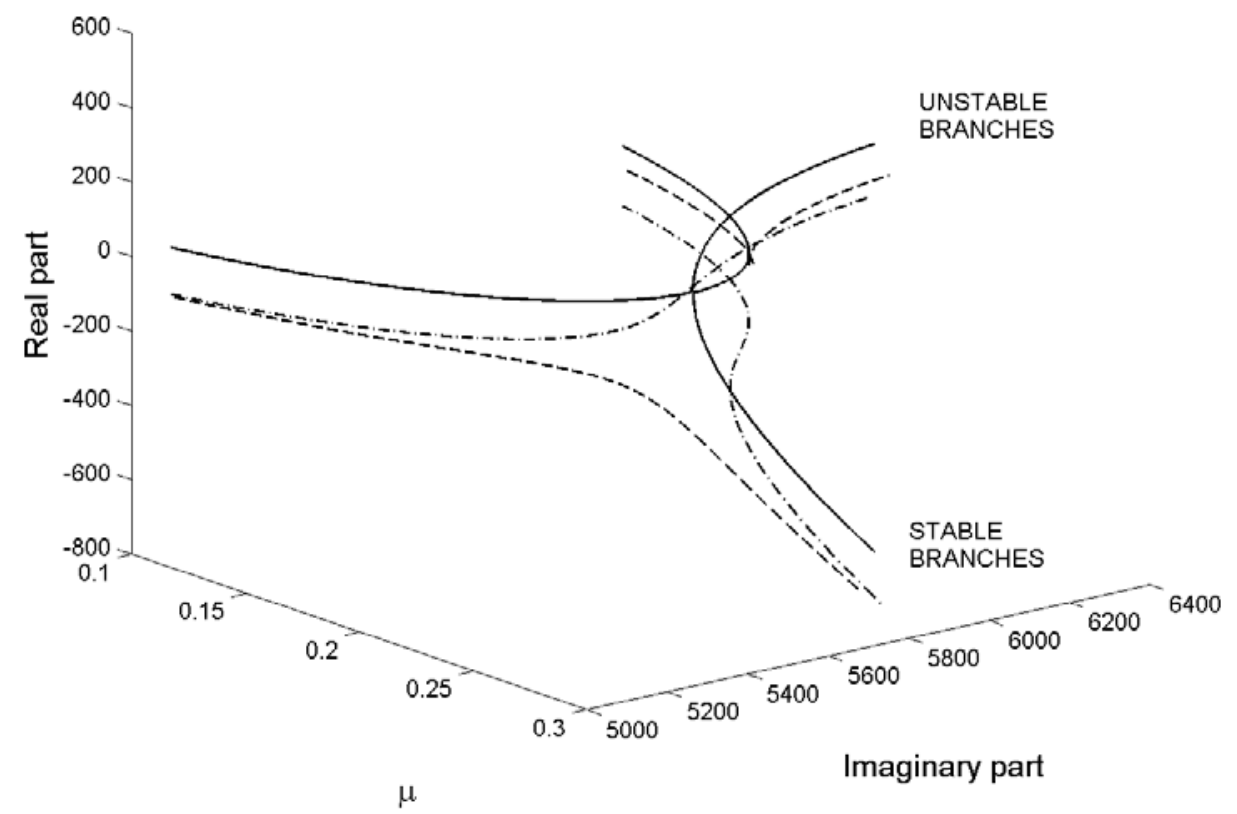

Figure 3 : Evolution of the stable and unstable branches against the friction coefficient $\mu$ for various proportional structural damping

$$
\left(-\eta_{1}=\eta_{2}=0----\eta_{1}=0.02, \eta_{2}=0.05-\cdot-\cdot-\eta_{1}=\eta_{2}=0.05\right)
$$




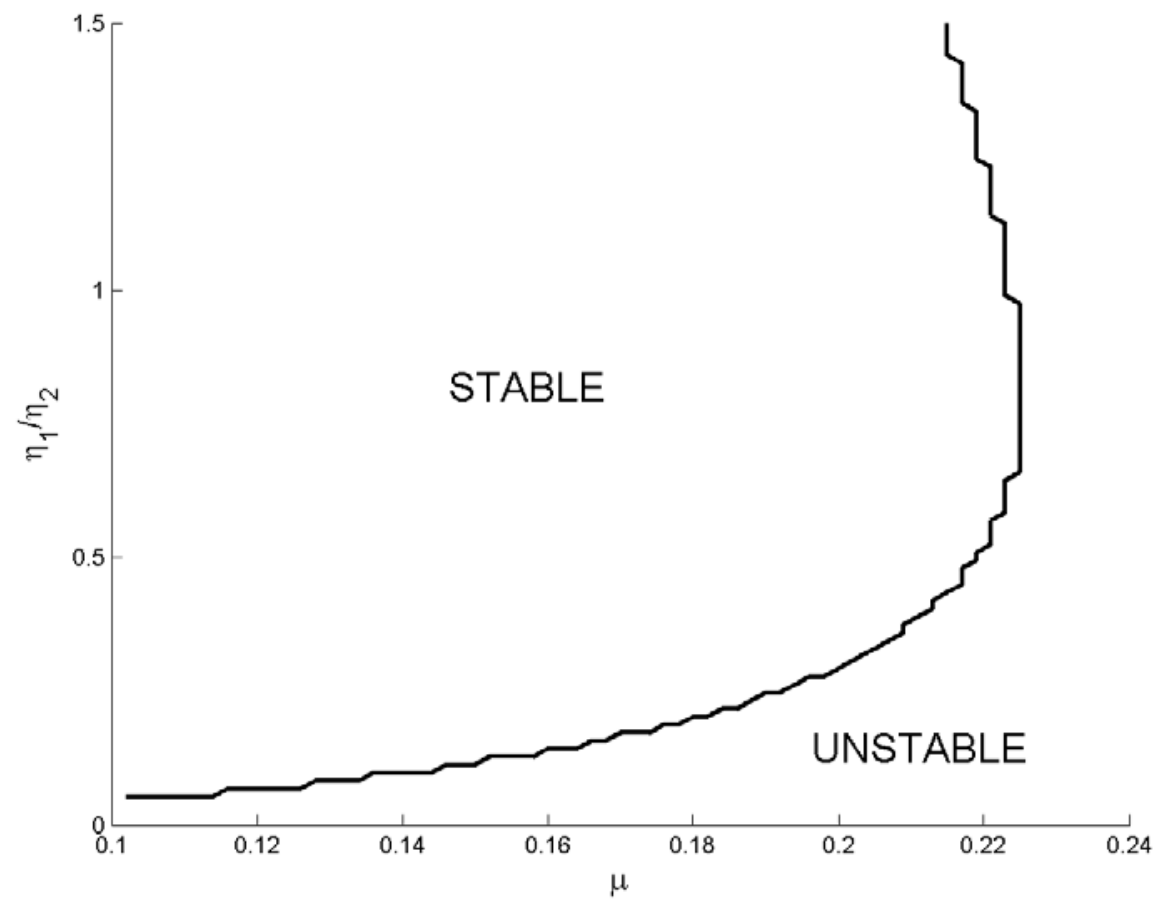

Figure 4 : Stable and unstable zones versus the damping ratio $\eta_{1} / \eta_{2}$ and the friction coefficient $\mu$ (with $\eta_{1}=0.02$ )

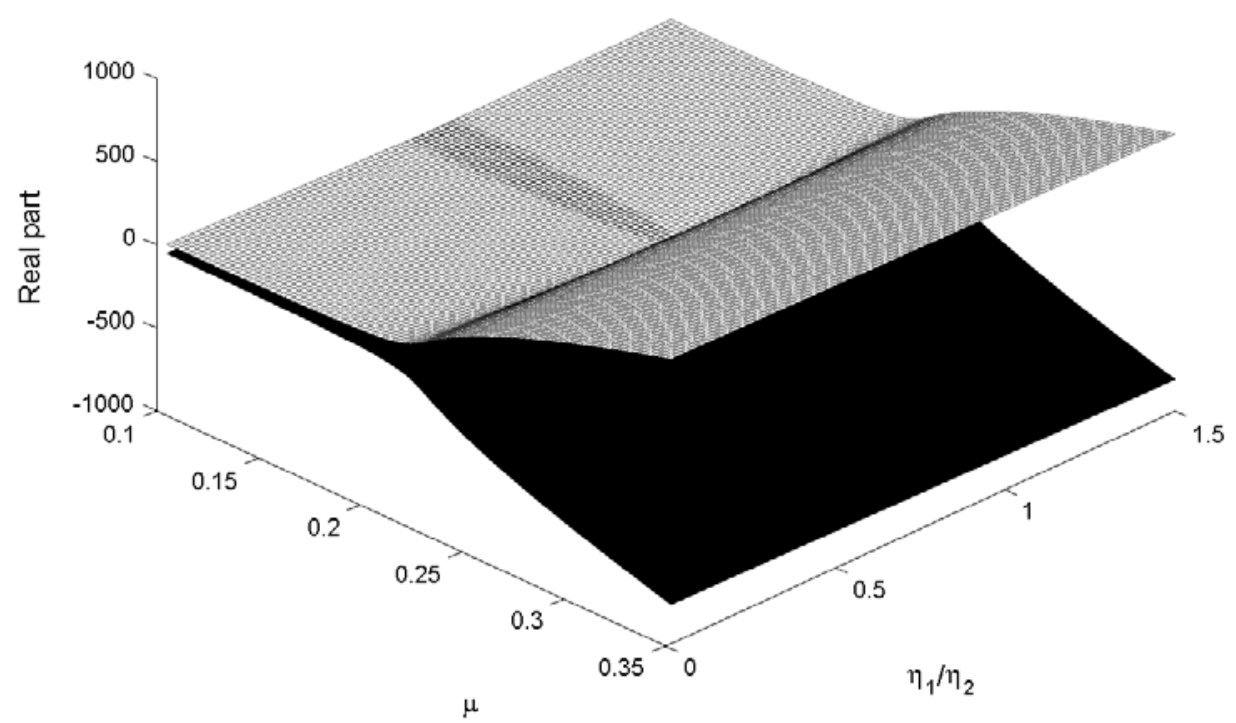

Figure 5 : Evolution of the real part of eigenvalues versus the damping ratio $\eta_{1} / \eta_{2}$ and the friction coefficient $\mu$ (with $\eta_{1}=0.02$ )

(black surface=stable branch, white surface=unstable branch) 


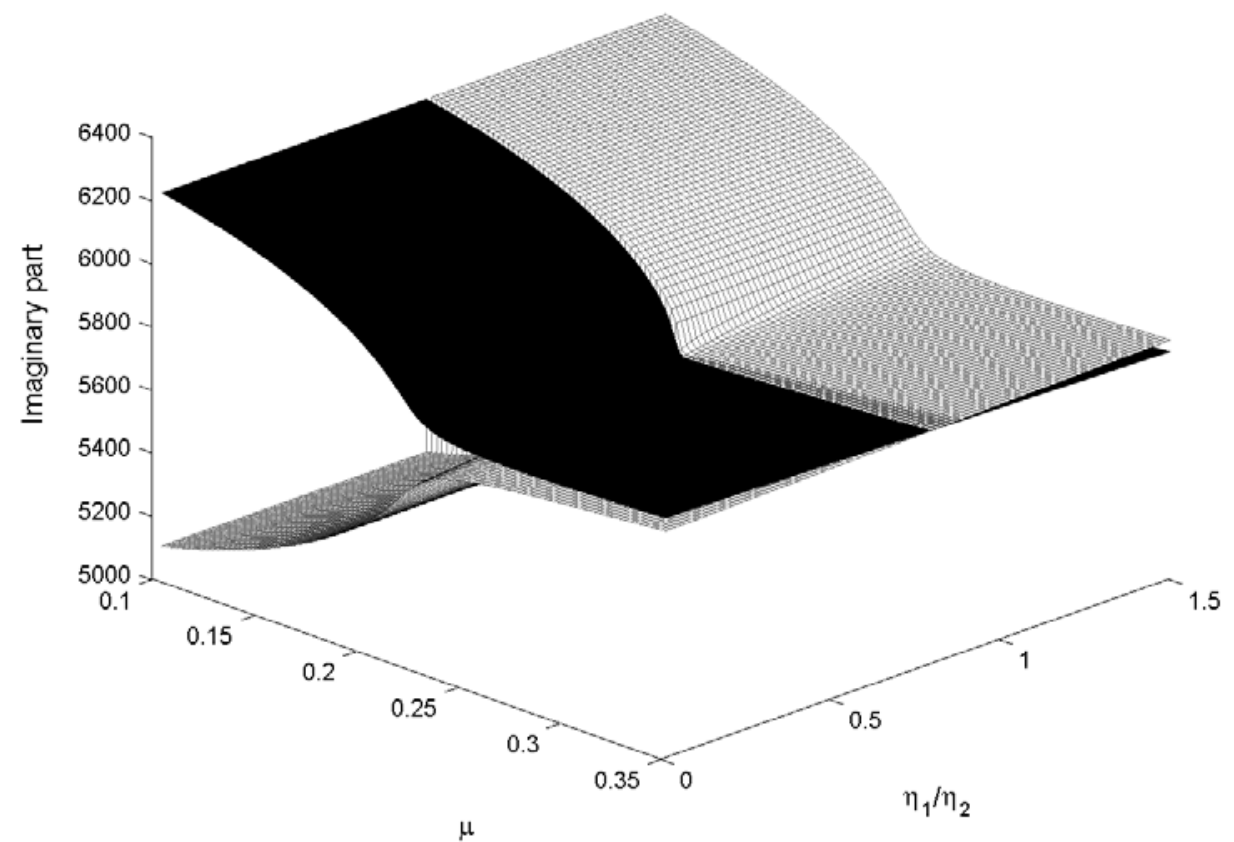

Figure 6 : Evolution of the imaginary part of eigenvalues versus the damping ratio $\eta_{1} / \eta_{2}$ and the friction coefficient $\mu$ (with $\eta_{1}=0.02$ )

(black surface=stable branch, white surface=unstable branch)
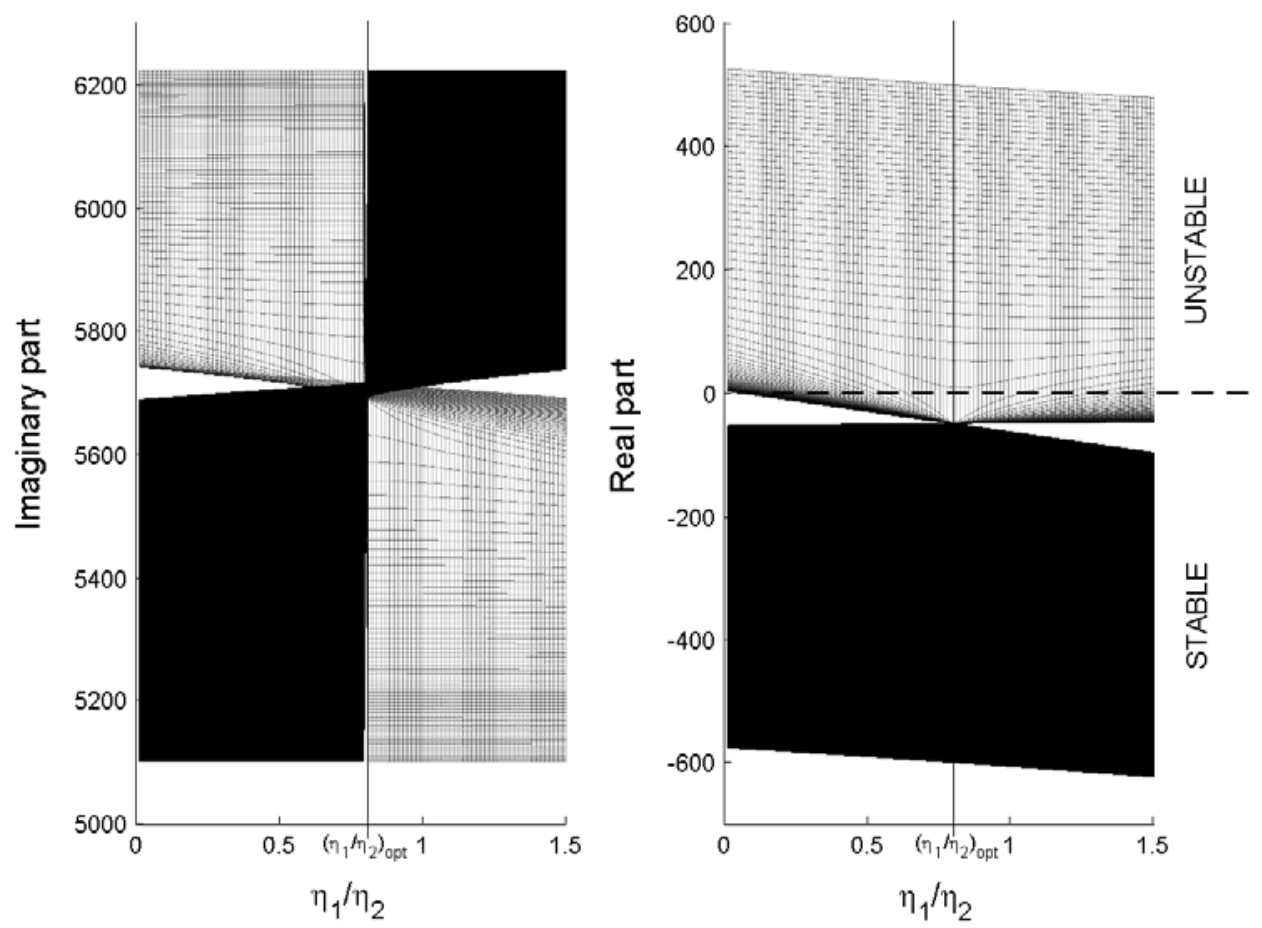

Figure 7 : Evolution of the real and imaginary part of eigenvalues versus the damping ratio $\eta_{1} / \eta_{2}$ (with $\eta_{1}=0.02$ )

(black surface=stable branch, white surface=unstable branch) 


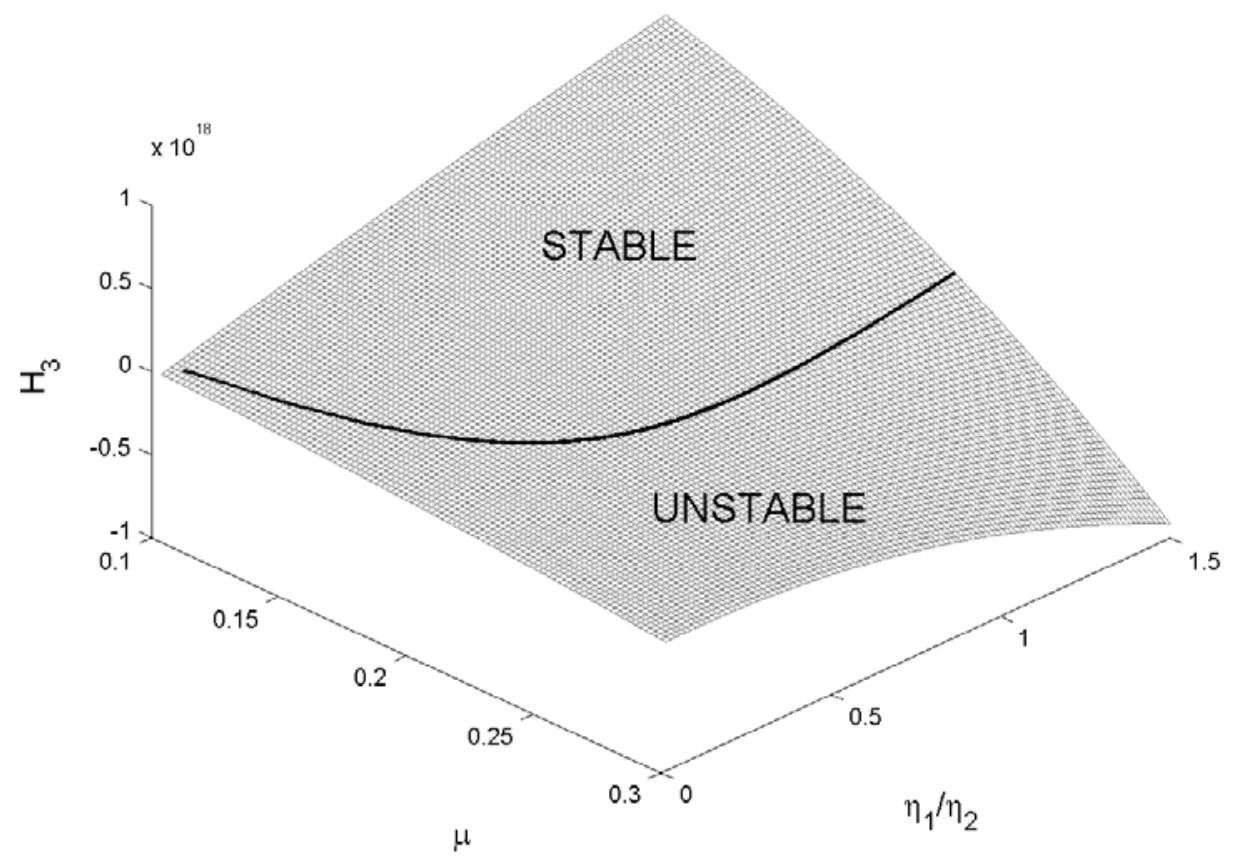

Figure 8 : Evolution of the routh hurwitz coefficient $H_{3}$ versus the friction coefficient $\mu$ and the damping ratio $\eta_{1} / \eta_{2}$ (with $\eta_{1}=0.02$ )

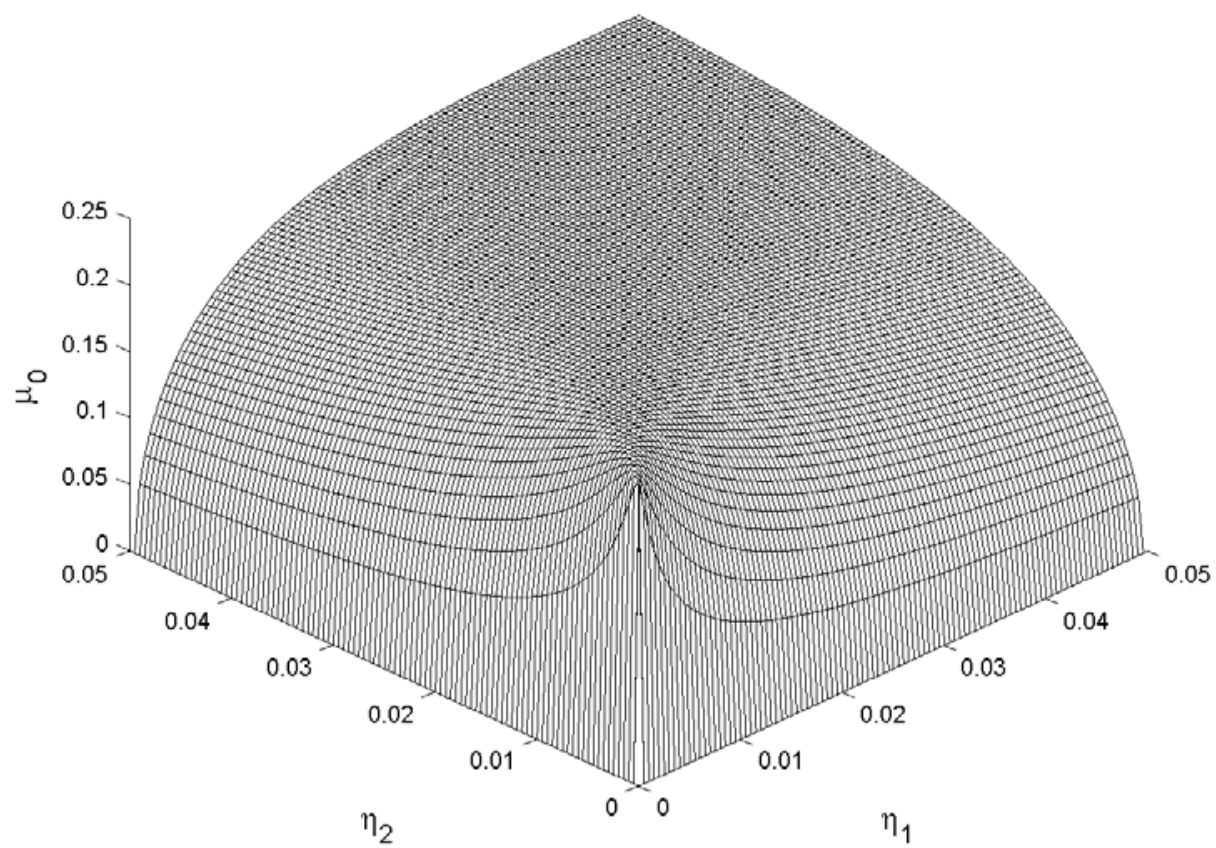

Figure 9 : Evolution of the Hopf bifurcation point $\mu_{0}$ versus the structural damping $\eta_{1}$ and $\eta_{2}$ 


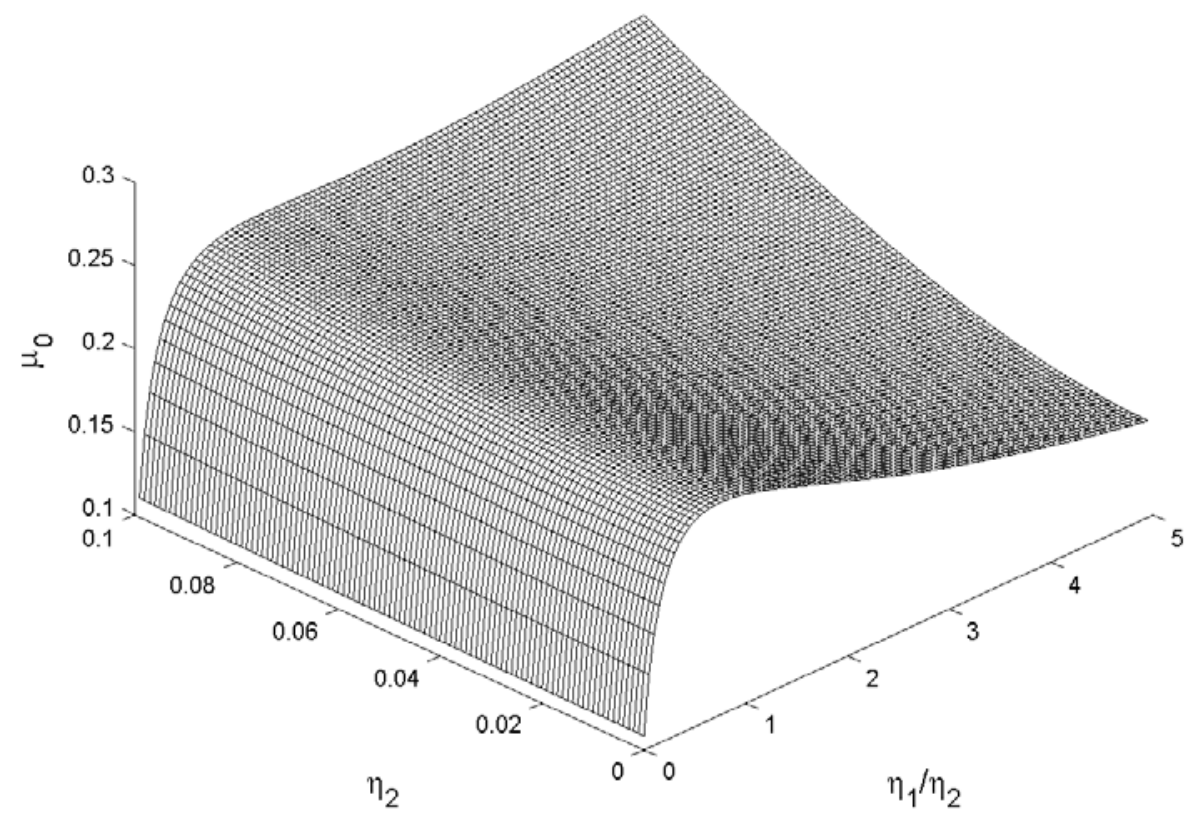

Figure 10 : Evolution of the Hopf bifurcation point $\mu_{0}$ versus the structural damping $\eta_{2}$ and damping ratio $\eta_{1} / \eta_{2}$

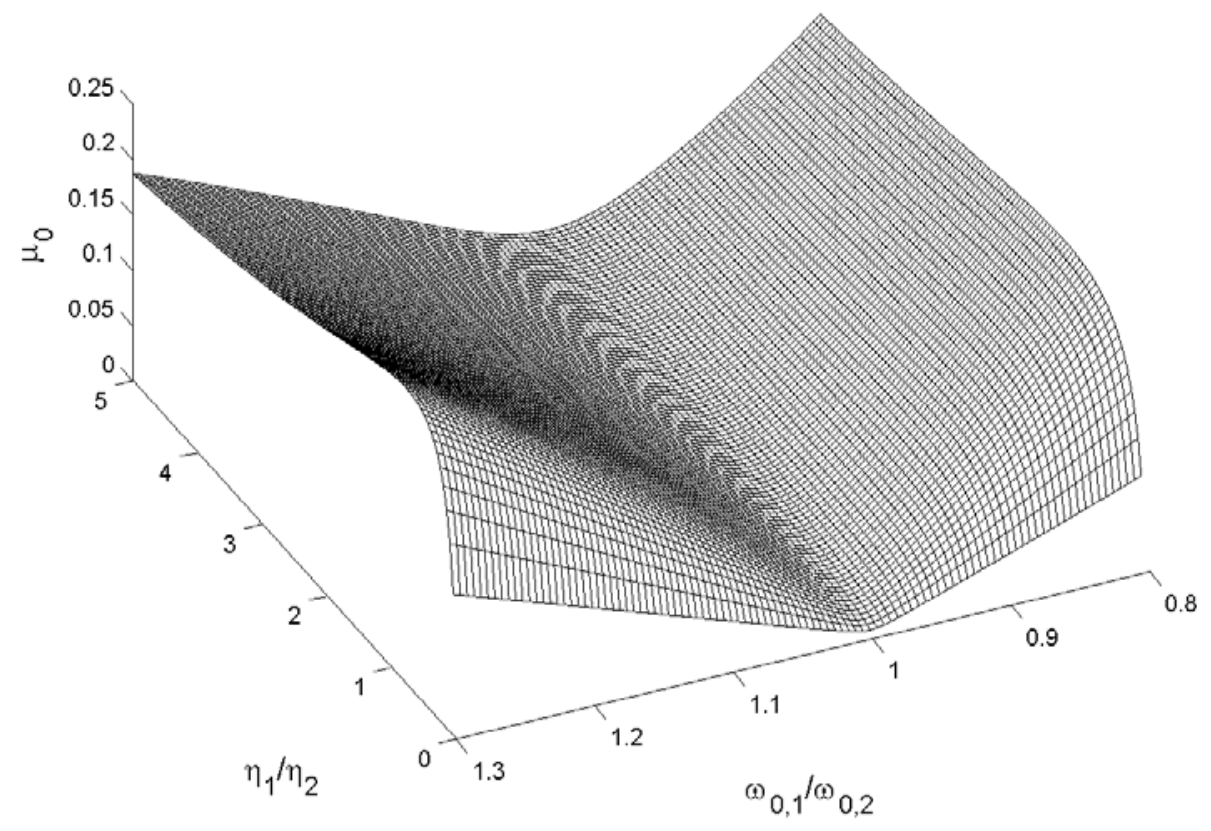

Figure 11 : Evolution of the Hopf bifurcation point $\mu_{0}$ versus the damping ratio $\eta_{1} / \eta_{2}$ and the pulsation ratio $\omega_{0,1} / \omega_{0,2}$ (with $\eta_{1}=0.02$ ) 


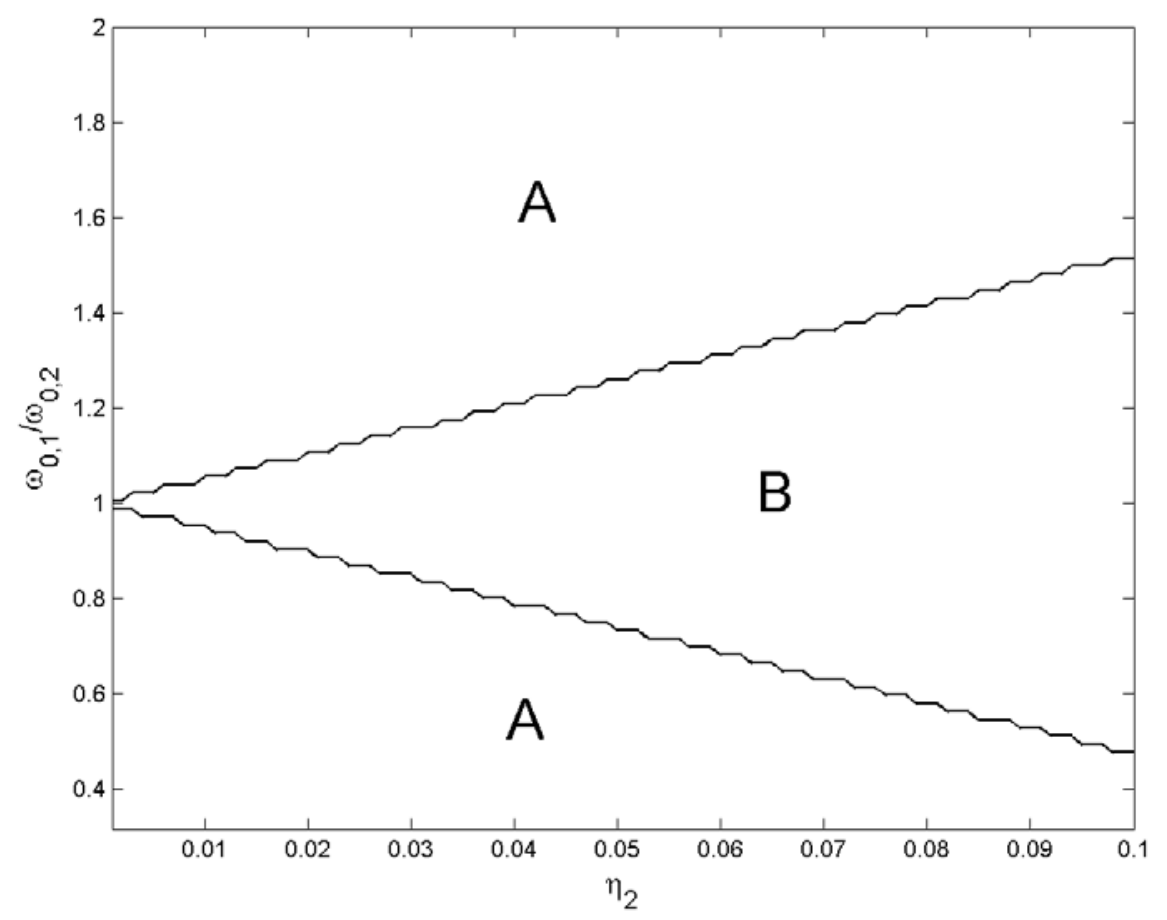

Figure 12 : Determination of the areas of existence of an optimal damping ratio $\eta_{1} / \eta_{2}$ (zones A: existence of an optimal damping ratio; zones B: no optimal damping ratio)

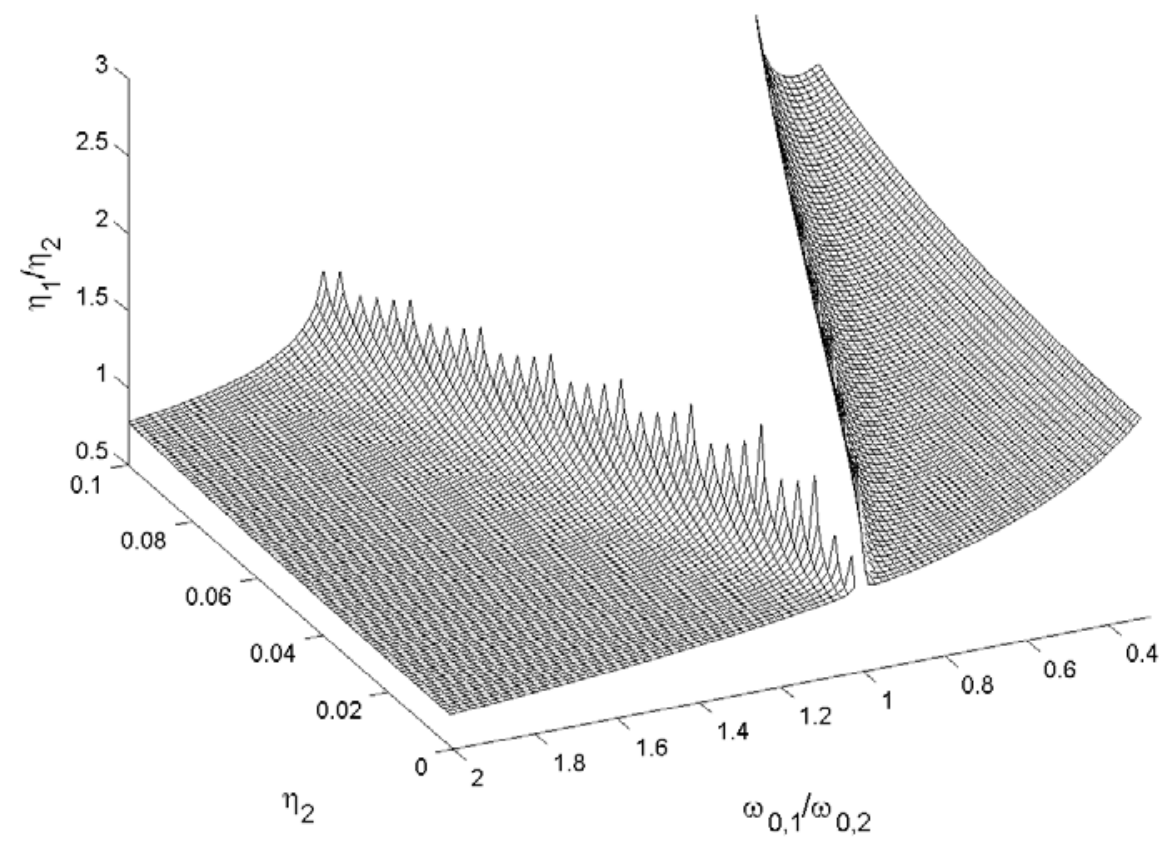

Figure 13 : Evolution of optimal damping ratio $\eta_{1} / \eta_{2}$ versus the damping $\eta_{2}$ and the pulsation ratio $\omega_{0,1} / \omega_{0,2}$ 


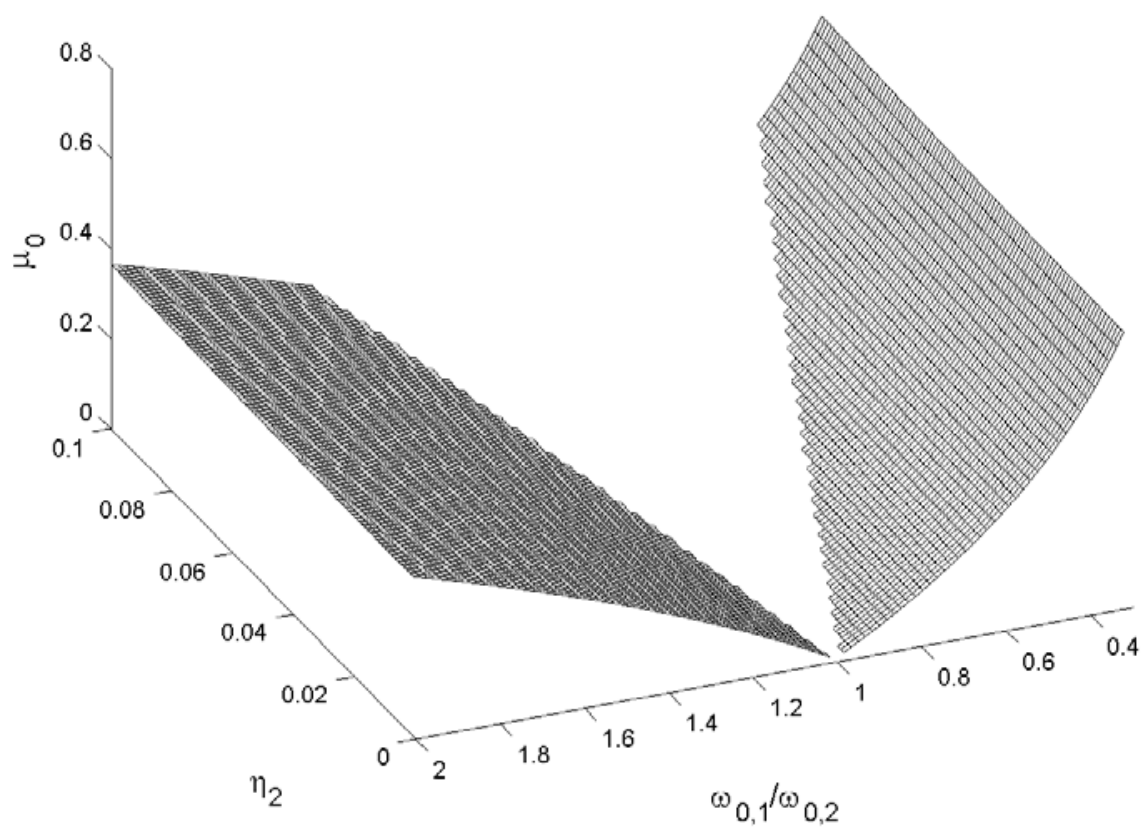

Figure 14 : Evolution of the Hopf bifurcation point $\mu_{0}$ versus the damping $\eta_{2}$ and the pulsation ratio $\omega_{0,1} / \omega_{0,2}$ for the optimal damping ratio $\eta_{1} / \eta_{2}$ 

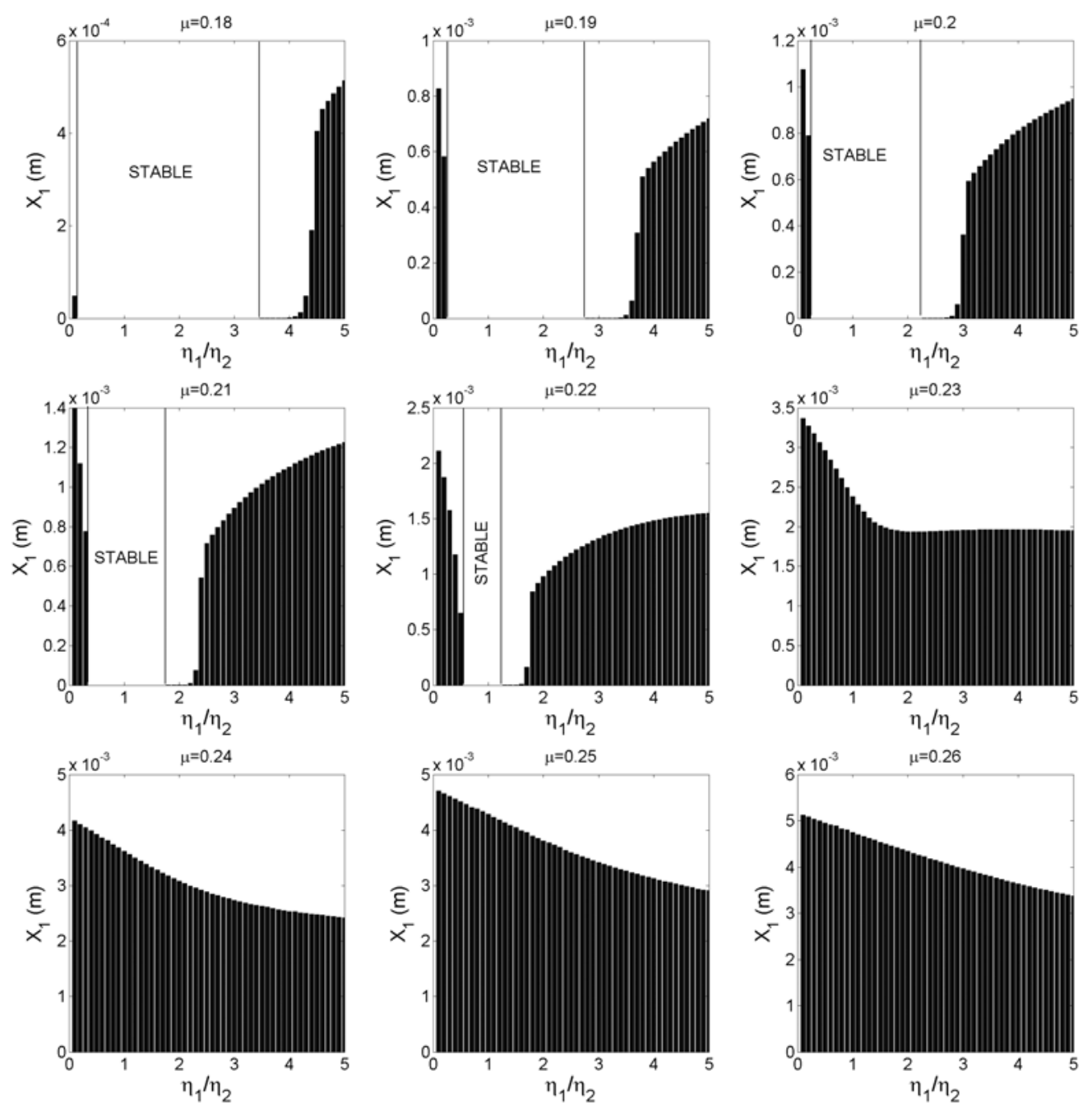

Figure 15 : maximal amplitudes $X_{1}$ for various friction coefficient and damping ratio $\eta_{1} / \eta_{2}$ (with $\eta_{1}=0.02$ ) 

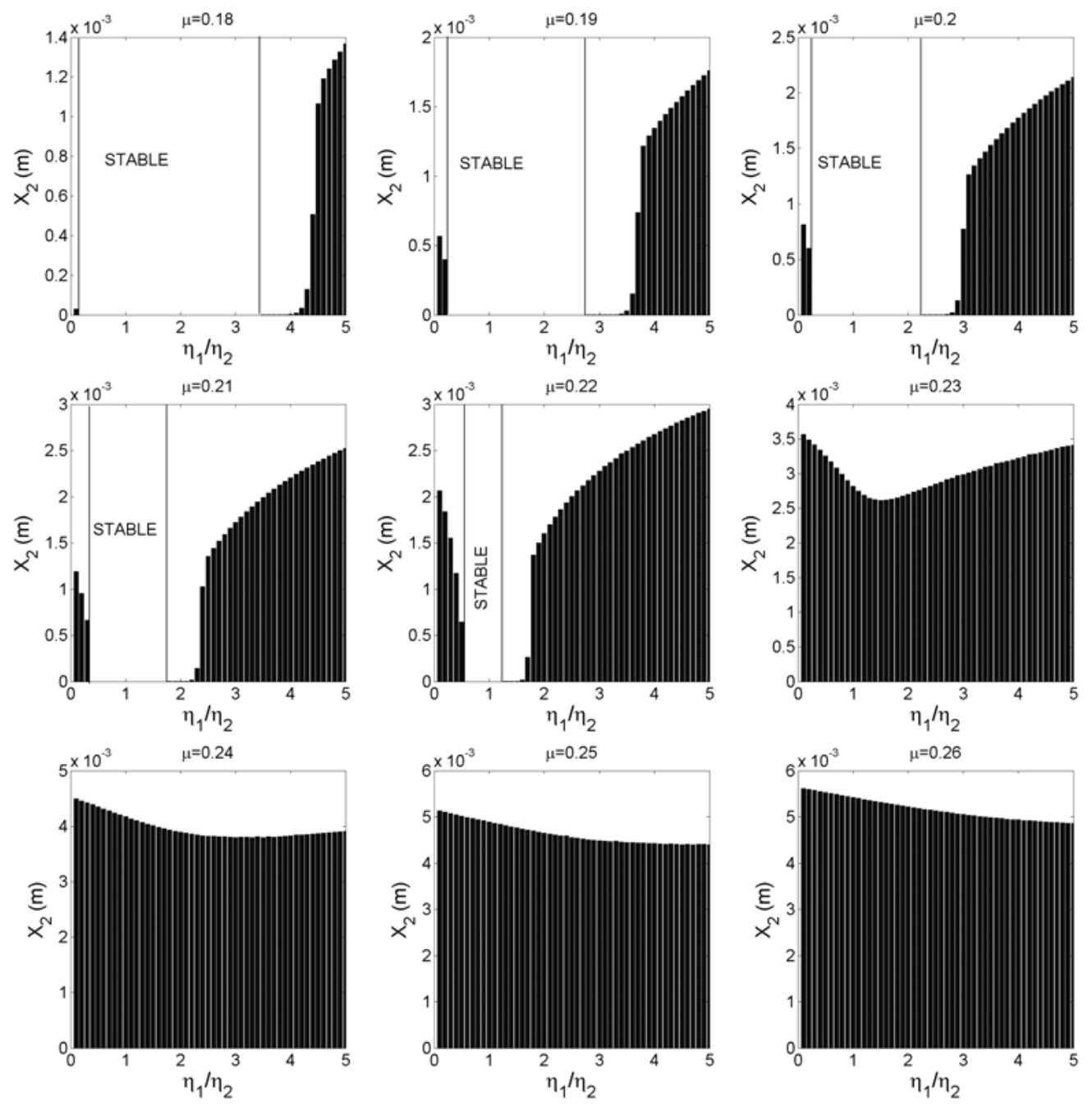

Figure 16 : maximal amplitudes $X_{2}$ for various friction coefficient and damping ratio $\eta_{1} / \eta_{2}$ (with $\eta_{1}=0.02$ ) 